\title{
The Comparison of Object Color Knowledge Between Congenitally Blind and Sighted People
}

\author{
Jie Feng ${ }^{1}$, Juan $\mathrm{Xu}^{2}$, Xinchun $\mathrm{Wu}^{3}$, * \\ ${ }^{1}$ Beijing Electronic Science and Technology Institute, Beijing, China \\ ${ }^{2}$ Special Education College, Beijing Union University, Beijing, China \\ ${ }^{3}$ Faculty of Psychology, Beijing Normal University, Beijing, China
}

\section{Email address:}

myonly88@126.com (Jie Feng), sftxujuan@buu.edu.cn (Juan Xu), xcwu@bnu.edu.cn (Xinchun Wu)

${ }^{*}$ Corresponding author

\section{To cite this article:}

Jie Feng, Juan Xu, Xinchun Wu. The Comparison of Object Color Knowledge Between Congenitally Blind and Sighted People. Science Innovation. Vol. 9, No. 1, 2021, pp. 12-18. doi: 10.11648/j.si.20210901.13

Received: January 25, 2021; Accepted: February 19, 2021; Published: March 4, 2021

\begin{abstract}
What role does perception play in human cognition? This is an important question in the research area of psychology. The current study aimed at exploring the influence of visual perceptual experience on human color cognition, by comparing the differences in behavioral and neuropsychological responses between congenital-blind and normal-sighted participants. The current study employed a semantic-priming paradigm, and presented prime-target word pairs to the participants randomly, such as, /bai2se4/ (white) -/xue3hua1/ (snow), or /bai2se4/ (white) - /mei2tan4/ (coal). All participants were instructed to decide whether the color term and the color of the object were matched or not. Results showed that (1) congenital-blind participants had acquired some color knowledge, but they were not as good as the sighted participants. (2) Mismatched color relationships induced significant N400 effects in both of the two groups, with a left hemisphere asymmetry. (3) However, compared with the sighted group, the blind group exhibited stronger N400 effects (i.e., larger amplitude of the difference waveform) and shorter N400 peak latencies. The current study suggests that congenital blindness have induced some cognitive and neuropsychology adaptive changes in blind people, and first-hand visual perceptual experience is important to color cognition but may be not prerequisite.
\end{abstract}

Keywords: Congenital Blindness, Object Color Knowledge, Color Cognition, N400

\section{先天盲人与明眼人颜色知识的对比研究}

冯杰 $^{1}$, 徐娟 ${ }^{2}$, 伍新春 ${ }^{*}$

1 北京电子科技学院, 北京, 中国

2 北京联合大学特殊教育学院, 北京, 中国

${ }^{3}$ 北京师范大学心理学部, 北京, 中国

邮箱

myonly88@126.com（冯杰），sftxujuan@buu.edu.cn（徐娟），xcwu@bnu.edu.cn（伍新春）

摘要: 个体的感觉经验对认知有何影响? 这是心理学领域一个非常重要的研究问题。本研究通过对比先天盲被试和明 眼被试颜色知识水平及其神经表征的差异, 进而探讨视觉经验与颜色认知之间的关系。研究采用语义启动范式, 向先 天盲被试和明眼被试随机呈现启动词-目标词词对（比如/白色-雪花/或/白色-煤炭/），被试需要依据常识来判断所听到 的两个词的颜色关系是否匹配。结果发现：（1）先天盲被试具有一定的颜色知识，但其水平低于明眼被试；（2）两 组被试中都有出现由颜色关系不匹配所诱发的N400效应, 且这种N400效应在左侧电极更强, 说明盲人被试和明眼被试 
大脑对颜色知识的表征均具有左半球优势; （3）但是, 先天盲组N400反应差异波的波幅比明眼组大, N400反应的潜 伏期比明眼组短。本研究说明, 先天视觉经验缺失的盲人在颜色认知及其神经生理表征方面均产生了一些适应性改变, 直接的视觉经验对个体颜色认知的形成具有重要作用但并非必要条件。

关键词: 先天盲, 物体颜色知识, 颜色认知, N400

\section{1. 前言}

感觉是较初级的心理过程, 语言、思维、情绪等则是 高级认知过程。个体的感觉与认知之间究竟有着什么样的 关系呢? 一些研究者认为, 感觉与认知是相互独立的心理 过程, 感觉知识以抽象符号的形式存储于概念层面, 并与 感觉表征相分离 [6-7,13,14]。但是, 更多的研究者认为, 认知与感觉并不是相互独立的, 认知以感觉体验为基础, 个体对周围世界的知识表征储存于感觉连接皮层 $[1,9$, $16]$ 。视觉是人类的一种重要感觉通道, 明眼人在日常生 活中有意无意地习得各种颜色知识。人们通过观察蔬菜的 颜色来判断其是否新鲜; 看到红灯停, 看到绿灯行。与明 眼人不同, 先天盲个体从一出生便丧失视觉, 颜色无形、 无味、无声, 视觉通道受阻的先天盲人无法通过眼睛来直 接感受各种各样的色彩。先天盲人和明眼人具有迥异的视 觉经验, 为研究者探究感觉 (本研究主要关注视觉) 与认 知之间的关系提供了非常宝贵的研究模型。本研究主要通 过对比先天盲人和明眼人的颜色知识水平及其神经表征 的差异, 进而探讨视觉经验与颜色认知之间的关系。

以往对盲人基本颜色认知的研究发现, 盲人开始使 用颜色词的时间与同龄明眼人相似。有研究报告一个4 岁的盲童可以指出一些物体的颜色特征 [4], 8-13岁的盲 童能够说出大部分物体的颜色 [10]。在颜色相似性判断任 务中, 盲人与明眼被试的表现模式也具有高度相似性 $[8$, $12 ， 15]$ 。我国研究者张积家及其同事[19]对盲童和明眼 儿童的颜色概念进行了对比研究, 结果显示, 中国盲童 颜色概念的通过顺序与同龄明眼儿童有所不同, 后天盲 儿童对“白”和“紫”的通过率 (即能够说出具有某种颜色 的物体的比例）显著高于先天盲儿童, 中学盲童对“棕” 的通过率显著高于小学盲童; 在颜色词的分类上, 盲童 和正常儿童都有彩色/非彩色的维度, 但明眼儿童还有暖 色/冷色维度, 盲童的分类维度中有实物色/背景色的维度。 此外, 时琴琴[18]发现, 初中阶段, 盲童在不包含红、绿、 蓝的八种基本颜色的通过率显著低于视力正常儿童, 两 组儿童在对颜色的分类维度上也有所差异。这些研究结 果说明, 盲童具有一定颜色概念, 他们可能是通过语言 描述（口语或盲文）来获得一定颜色相关知识; 但盲童 颜色概念的获得时间和分类与明眼儿童有一定区别, 说 明直接的视觉感知经验的缺失对盲童颜色概念的获得具 有一定影响作用。个体在儿童期的认识和能力处于快速 发展变化阶段, 盲童与明眼儿童颜色认知上所表现出的 差异可能是一种暂时的落后, 也可能是一种质的差异。

比如, Pérez-Pereira和Conti-Ramsden[11]认为视觉输入缺 失所造成的概念缺陷可以通过口语学习和推理而获得补 偿; 语言作为一种补偿学习工具, 可以促进盲童世界知
识的获得。成年人的认知能力的发展相对稳定, 因此, 为了更清楚地说明视觉经验缺失对盲人颜色认知的影响, 本研究以先天盲成人为研究对象, 比较先天盲被试和明 眼被试所获得的颜色知识的差异。明眼人既可以通过直 接的感知来获取颜色知识, 也可通过外界的语言描述 (包 括书面语和口语) 来进行学习, 而视觉通道受阻的先天 盲人只能通过语言描述来获得颜色知识。对先天盲人和 明眼人颜色知识的对比研究可以帮助我们认识视觉经验 缺失对个体颜色知识获得的影响。

本研究使用事件相关电位 (Event-related potential, ERP）技术，事件相关电位具有较高的时间分辨率，可以 对认知加工的过程进行连续测量; 并且, 对于不同水平的 加工分析有不同的科学敏感的指标。其中N400是语义加工 中常用的ERP指标, 它表征语义记忆提取和/或整合 $[2,3$, $5]$ 。N 400 是一个广泛分布的负的ERP成分, 它在听觉或视 觉呈现的词出现 $200-300 \mathrm{~ms}$ 后开始出现, 并在 $400 \mathrm{~ms}$ 左右 达到峰值; 这种由不同实验条件所诱发的N 400 反应波幅的 差异或变化称为 $\mathrm{N} 400$ 效应 $[2,3]$ 。语义启动范式是 $\mathrm{N} 400$ 研究常用的实验范式[5], 即在目标词出现之前, 向被试呈 现与目标词相关或不相关的启动词（比如“咖啡-茶”和“椅 子-茶”）。无关条件所诱发的 $\mathrm{N} 400$ 反应的波幅比相关条件 所激发的 $\mathrm{N} 400$ 反应的波幅更大, 这两种实验条件所诱发的 $\mathrm{N} 400$ 反应波幅的差异即为 $\mathrm{N} 400$ 效应。

本研究采用语义启动范式, 向先天盲被试和年龄匹配 的明眼被试呈现颜色匹配词对 (比如/白色-雪花/) 和颜色 不匹配词对 (比如/白色-煤炭/), 让被试依据常识来判断 所听到的目标词的颜色与前面的颜色词是否匹配。在判断 过程中, 同时记录被试的行为反应和脑电信号变化, 并对 两组被试 $\mathrm{N} 400$ 反应的波幅、潜伏期以及行为反应指标进行 分析和比较, 探讨视觉经验缺失对个体颜色知识水平及其 神经表征的影响。

\section{2. 研究方法}

\section{1. 被试}

参与本实验的盲人组有盲人在校大学生 17 人 (包括 11 名男生和6名女生)，平均年龄为 20.94 岁 $(S D=1.48)$ 。 所有盲人被试均为先天盲, 他们平均开始学习盲文的时间 是7.5岁 $(S D=1.52)$ 。明眼组包括11名男生和10名女生, 平均年龄为 21.33 岁 $(S D=1.53)$, 视力或矫正视力正常, 且颜色视力正常。两组被试年龄匹配 $(t(36)=0.80, p=$ $0.430 ）$, 所有被试均为右利手, 无听力损伤, 无精神或 神经疾病。本研究获得每位被试知情同意, 并在测试结束 后给与被试一定报酬。 


\section{2. 实验材料}

本研究使用 174 对双音节汉语词作为听力材料, 采用 启动范式, 向被试听觉呈现启动词-目标词词对。匹配条 件下, 作为启动词的颜色词与作为目标词的物体正常状态 下的颜色是一致的, 比如/白色-雪花/; 不匹配条件下, 颜 色词与目标词物体正常状态下的颜色是不一致的, 比如/ 白色-煤炭/。所有听觉刺激材料由一名以汉语为母语的成 年女性录制。录音采样率为 $44.1 \mathrm{kHz}$, 使用Praat做标准化 处理, 每个词的音强标准化为 $70 \mathrm{~dB}$ 、音长标准化为 $600 \mathrm{~ms}$ 。

\section{3. 实验过程}

\subsection{1. 测试流程}

对全部174个词对进行伪随机排序, 其中4对词用作练 习, 其余 170 个词对分为 2 个测试组, 每个测试组中有 85 个试次（词对），使用E-prime2.0编写和呈现测试程序。 每个试次开始时, 首先出现一个 $600 \mathrm{~Hz}$ 的纯音 $(500 \mathrm{~ms})$, 之后出现一个作为启动词的颜色词 $(600 \mathrm{~ms})$, 在一个 $500-800 \mathrm{~ms}$ 的随机空白间隔后出现目标词 $(600 \mathrm{~ms})$ 。被试 需要在目标词出现之后的 $3000 \mathrm{~ms}$ 内尽量快速而准确的判 断启动词与目标词所表示的颜色是否匹配, 判断依据一般 常识并且只考虑事物正常状态下的颜色, 比如/红色-苹果/ 是匹配的, /黄色-苹果/也是匹配的, 而/黑色-苹果/则是不 匹配的。匹配按F键，不匹配按J键。完成该任务需要 12 分 钟左右, 每个测试组结束即可休息, 休息时长由被试自己 控制。为了避免使用耳机可能带来的额外电阻的干扰, 本 研究使用音箱来呈现语音刺激。两个音箱放在距被试1米 远的前方左右各一个, 各与被试成 $45^{\circ}$ 角。

\subsection{2. 数据收集}

本研究使用Neuroscan 4.5 和SynAmps EEG放大器收集 脑电数据, 采样率为 $1000 \mathrm{~Hz}$, 带宽为 $0.05-100 \mathrm{~Hz}$ 。每个被
试佩戴一顶Quick-Cap64导电极帽, 帽子电极依据国际 10-20系统分布于全脑。使用系统默认的参考电极, 垂直 眼电在左眼上下各一个, 水平眼电在左右两侧太阳穴各一 个, 每个电极上的电阻控制在不超过 $5 \mathrm{k} \Omega$ 。在脑电实验过 程中, 同时收集脑电信号和行为反应数据。

\section{4. 数据分析}

离线脑电数据处理, 首先, 转换参考电极, 用 $\mathrm{M} 1$ 和 M2的平均电位作为参考, 并且只分析被试进行正确反应 的试次; 使用Neuroscan提供的线性回归方程对眼电进行 校正; 滤波使用 $30 \mathrm{~Hz}$ 、Zero-phase和 $24 \mathrm{~dB} /$ octave的低通滤 波; 以刺激出现前 $200 \mathrm{~ms}$ 为基线、刺激出现后 $1000 \mathrm{~ms}$ 时间 段对数据进行分段; 基线校正后, 去除任何电极上电位变 化超出 $\pm 100 \mu \mathrm{V}$ 的trail; 最后, 将每个被试两种实验条件下 剩余的trail分别进行叠加平均。

本研究主要关注N400成分。对本实验中的波形图 (见 图1) 进行观察, 发现在目标刺激出现后 $300 \mathrm{~ms}$ 左右开始出 现一个负波, 并在 $550 \mathrm{~ms}$ 左右达到峰值, 该成分这可能判 断为是 $\mathrm{N} 400$ 反应。根据总体叠加平均的波形图 (图1) 和 地形图 (图2), 选取300-800ms作为分析 $\mathrm{N} 400$ 的时间窗, 提取该时间窗内的平均波幅和峰值潜伏期。进入分析的电 极包括, 分布于左半球的 6 个电极 $(\mathrm{FC} 1 、 \mathrm{FC} 3 、 \mathrm{C} 1 、 \mathrm{C} 3$ 、 $\mathrm{CP} 1$ 和CP3) 和分布于右半球的 6 个电极 (FC2、FC4、C2、 $\mathrm{C} 4 、 \mathrm{CP} 2$ 和 CP4) 。以实验条件（匹配和不匹配）和半球 分布 (左侧和右侧) 为被试内变量, 以分组 (盲人组和明 眼人组) 为被试间变量, 使用 $2 \times 2 \times 2$ 三因素方差分析对 N400反应的平均波幅和峰值潜伏期分别进行统计分析。对 于行为反应的正确率和反应时, 以实验条件 (匹配和不匹 配) 为被试内变量, 分组 (包括盲人组和明眼组) 为被试 间变量, 使用两 $2 \times 2$ 因素方差分析, 比较两组被试不同实 验条件下行为反应的差异。超过正负三个标准差的数据作 为缺失值处理。

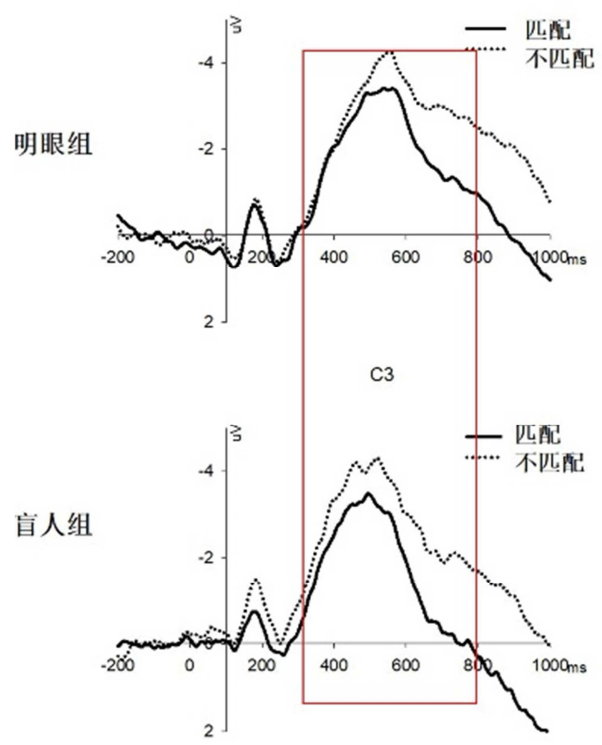

C4
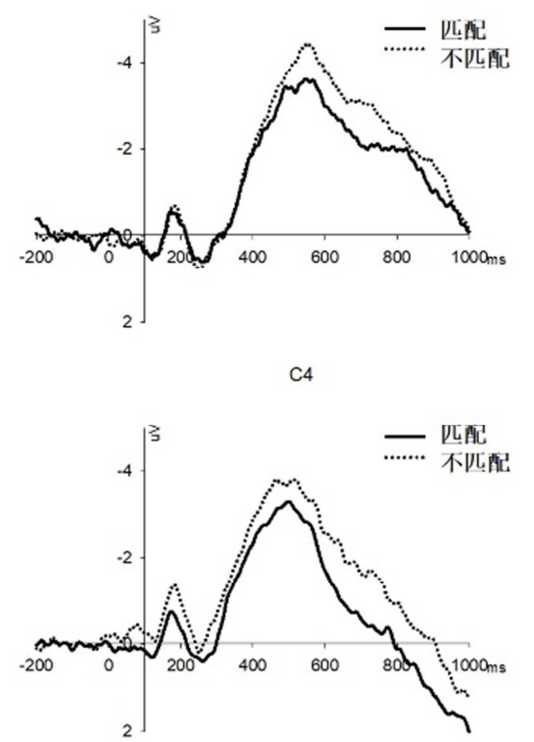

注: 矩形框所示为N400分析时间窗300-800ms。

图1 明眼组和盲人组N400反应波形图。 


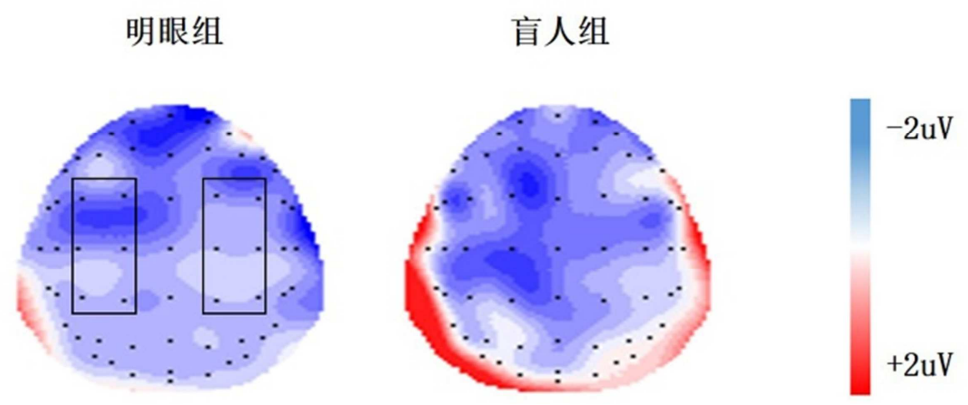

注: 矩形框表示选择分析的电极, 左侧电极 FC1、FC3、C1、C3、CP1和CP3, 右侧电极FC2、FC4、C2、C4、CP2和CP4。

图2 明眼组和盲人组 $N 400$ 效应地形图。

\section{3. 实验结果}

\section{1. 行为结果}

对正确率 (ACC) 的两因素方差分析 (实验条件 $\times$ 分 组) 结果显示, 分组主效应显著 $(F(1,36)=20.47, p<0.001$, $\left.\eta^{2}=0.36\right)$, 明眼被试的正确率高于畺人被试的正确率 (如 图3a); 实验条件主效应不显著 $(F(1,36)=1.53, p=0.224)$, 实验条件 $\times$ 分组交互作用也不显著 $(F(1,36)=1.12, p=$
0.297）。反应时（RT）的分析结果发现, 实验条件主效 应显著 $\left(F(1,36)=19.84, p<0.001, \eta^{2}=0.36\right)$, 实验条 件 $\times$ 分组交互作用显著 $\left(F(1,36)=6.19, p=0.018, \eta^{2}=\right.$ $0.15)$, 分组主效应不显著 $(F(1,36)=0.76, p=0.390)$ 。 使用Bonferroni多重矫正的简单效应分析结果显示, 盲人 被试对不匹配词对进行判断的反应时长于对匹配词对进 行判断的反应时 $(F(1,36)=21.80, p<0.001)$ (如图3b)， 明眼被试两种条件下的反应时无显著条件间差异 $(F(1,36)$ $=2.16, p=0.150$ )。

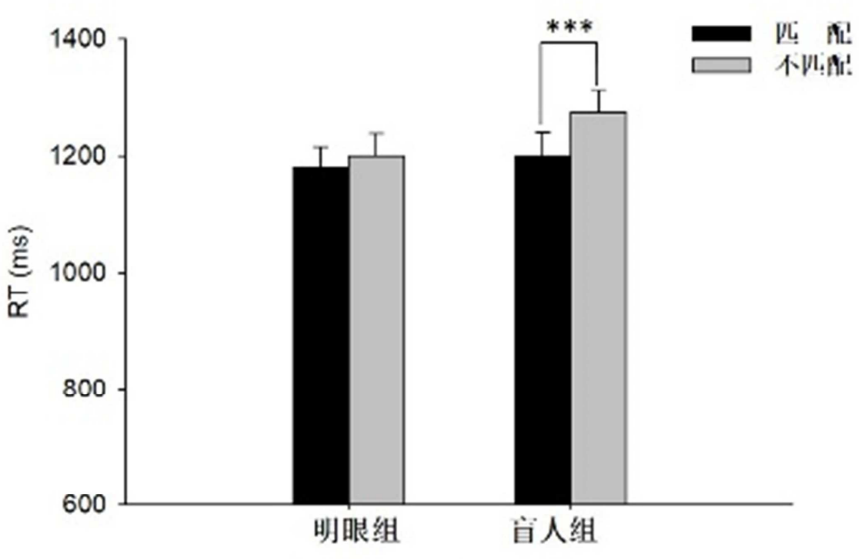

b.

注: $* * *$ 表示 $p<0.001$ 。

图3 明眼组和盲人组物体颜色知识判断的正确率 $\mathrm{ACC}$ 和反应时 RT。

\section{2. 脑电结果}

\subsubsection{N400反应波幅}

对 $\mathrm{N} 400$ 反应平均波幅的三因素方差分析（实验条件 $\times$ 半球分布 $\times$ 分组) 发现, 实验条件主效应显著 $(F(1,213)=$ 97.71, $\left.p<0.001, \eta^{2}=0.31\right)$, 实验条件 $\times$ 分组交互作用 显著 $\left(F(1,213)=21.01, p<0.001, \eta^{2}=0.09\right)$, 实验条 件 $\times$ 半球分布交互作用也显著 $(F(1,213)=25.12, p<0.001$, $\left.\eta^{2}=0.11\right)$ 。半球分布主效应不显著 $(F(1,213)=0.00, p$ $=0.984)$, 分组主效应不显著 $(F(1,213)=3.35, p=0.069)$, 半球分布 $\times$ 分组交互作用不显著 $(F(1,213)=1.85, p=$ $0.175)$, 三因素交互作用也不显著 $(F(1,213)=0.71, p=$ $0.400)$ 。
对实验条件 $\times$ 分组两因素交互作用进行简单效应分析, 结果显示, 明眼组中, 不匹配条件下N400反应的平均波幅 大于匹配条件下 $N 400$ 反应的平均波幅 $(F(1,437)=26.40$, $p<0.001)$; 盲人组中，不匹配条件下 $N 400 反$ 应的平均波 幅也大于匹配条件下的 $\mathrm{N} 400$ 反应平均波幅 $(F(1,437)=$ $151.49, p<0.001)$ 。匹配条件下，明眼组被试N400反应 的平均波幅大于盲人组被试 $\mathrm{N} 400$ 反应的平均波幅（ $F(1$, $437)=18.13, p<0.001)$; 不匹配条件下，两组被试N400 反应平均波幅大小无显著组间差异 $(F(1,437)=0.10, p=$ 0.759）。

对实验条件 $\times$ 半球分布两因素交互作用进行简单效应 分析, 结果发现, 左侧电极上, 不匹配条件下的N400波幅 大于匹配条件下的N400波幅 $(F(1,213)=17.10, p<0.001)$; 右侧电极上, 不匹配条件下的N400波幅也大于匹配条件下 
的 $\mathrm{N} 400$ 波幅 $(F(1,213)=16.84, p<0.001)$ 。匹配条件下， 左右两侧电极上 $\mathrm{N} 400$ 反应的平均波幅无显著半球差异 $(F(1,213)=0.92, p=0.340)$; 不匹配条件下, 两侧电 极上 $\mathrm{N} 400$ 平均波幅大小的差异也不显著 $(F(1,213)=2.60$, $p=0.108$ ）。

本研究进一步用不匹配条件下被试的N400反应减去 匹配条件下的N400反应, 得到N400反应的差异波, 该差 异波表示被试对不匹配刺激和匹配刺激的电生理反应的 差异, 即 $N 400$ 效应。使用 $2 \times 2$ 两因素方差分析, 以半球 分布 (左侧和右侧) 为被试内变量, 以分组 (明眼组和 盲人组) 为被试间变量, 对两组被试N400反应差异波 300-800ms内的平均波幅进行分析。两因素方差分析结果 显示, 半球分布主效应显著 $(F(1,213)=25.12, p<0.001$, $\left.\eta^{2}=0.11\right)$, 左侧电极上的 4400 反应差异波波幅大于右 侧电极上的 $\mathrm{N} 400$ 反应差异波波幅（如图4）; 分组主效应 显著 $\left(F(1,213)=21.01, p<0.001, \eta^{2}=0.09\right)$, 盲人组 $\mathrm{N} 400$ 反应差异波波幅大于明眼组N400反应差异波波幅

(如图4); 半球分布 $\times$ 分组交互作用不显著 $(F(1,213)=$ $0.71, p=0.400$ )。

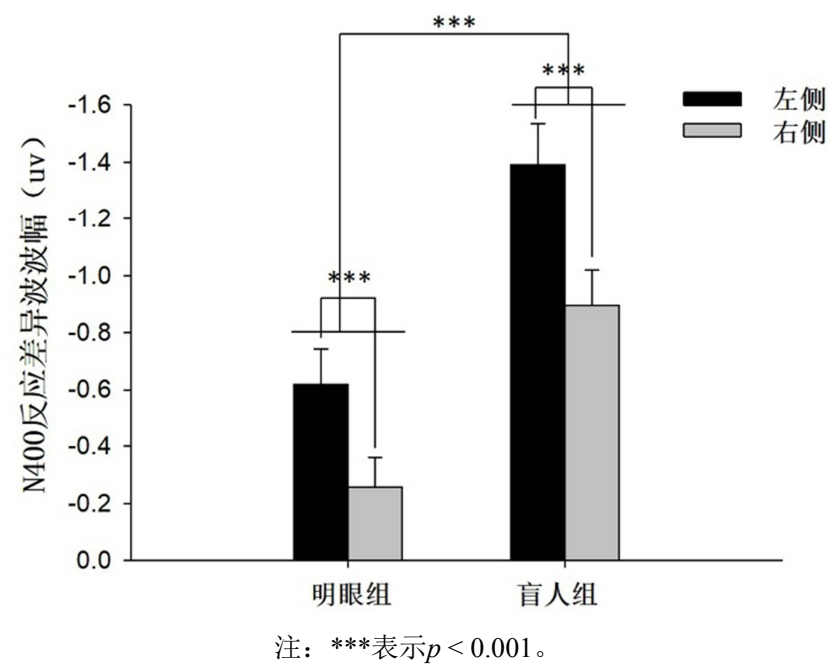

图4 盲人组和明眼组 $\mathrm{N} 400$ 反应差异波平均波幅 $(\mathrm{uV})$ 。

\subsubsection{N400反应潜伏期}

对 $\mathrm{N} 400$ 反应峰值潜伏期做实验条件 $\times$ 半球分布 $\times$ 分组 三因素方差分析, 结果显示, 实验条件主效应显著 $(F(1$, $\left.218)=39.48, p<0.001, \eta^{2}=0.15\right)$ ，半球分布主效应显 著 $\left(F(1,218)=8.78, p=0.003, \eta^{2}=0.04\right)$, 分组主效应 显著 $\left(F(1,218)=23.21, p<0.001, \eta^{2}=0.10\right)$ ，半球分 布 $\times$ 分组两因素交互作用显著 $(F(1,218)=4.30, p=0.039$, $\left.\eta^{2}=0.02\right)$, 三因素交互作用显著 $(F(1,218)=12.15, p=$ $\left.0.001, \eta^{2}=0.05\right)$ 。实验条件 $\times$ 分组两因素交互作用不显 著 $\left(F(1,218)=0.02, p=0.878, \eta^{2}=0.00\right)$, 半球分布 $\times$ 实验条件两因素交互作用也不显著 $(F(1,218)=2.23, p=$ $0.137, \eta^{2}=0.01$ )。

对实验条件 $\times$ 半球分布 $\times$ 分组三因素交互作用进行简单 简单效应分析, 结果显示, 左侧电极上匹配条件下, 盲人 组N400潜伏期小于明眼组N400潜伏期 $(F(1,218)=6.29, p$ $=0.013 ）$ ；左侧电极不匹配条件下，盲人组 $N 400$ 潜伏期小 于明眼组 $N 400$ 潜伏期 $(F(1,218)=18.69, p<0.001)$; 右 侧电极匹配条件下, 盲人组 $N 400$ 潜伏期小于明眼组N400潜 伏期 $(F(1,218)=26.81, p<0.001)$; 右侧电极不匹配条 件下, 盲人组 $N 400$ 潜伏期也小于明眼组N400潜伏期（ $F(1$, $218)=16.40, p<0.001)$ 。明眼组左侧电极上，不匹配条 件下的N400潜伏期大于匹配条件下的N400潜伏期（ $F(1$, $218)=39.41, p<0.001)$; 明眼组右侧电极上, 两种实验 条件下的N400潜伏期差异不显著 $(F(1,218)=1.45, p=$ $0.230 ）$; 盲人组左侧电极上, 不匹配条件下的 $\mathrm{N} 400$ 潜伏期 大于匹配条件下的 $\mathrm{N} 400$ 潜伏期 $(F(1,218)=5.42, p=0.021)$; 盲人组右侧电极上, 不匹配条件下 $N 400$ 潜伏期大也大于匹 配条件下的潜伏期 $(F(1,218)=15.08, p<0.001)$ 。明眼 组匹配条件下, 左侧电极上的 $\mathrm{N} 400$ 潜伏期小于右侧电极上 的N400潜伏期 $(F(1,218)=26.12, p<0.001)$ ；明眼组不 匹配条件下, 左右两侧电极上的 N400潜伏期无显著半球差 异 $(F(1,218)=1.42, p=0.234)$; 盲人组匹配条件下, 左 右两侧电极上的 $\mathrm{N} 400$ 潜伏期差异不显著 $(F(1,218)=0.05$, $p=0.831$ ); 盲人组不匹配条件下, 左右两侧电极上的 $\mathrm{N} 400$ 潜伏期也无显著差异 $(F(1,218)=1.54, p=0.216) 。$

表1 明眼组和盲人组 $N 400 反$ 应潜伏期（ms）。

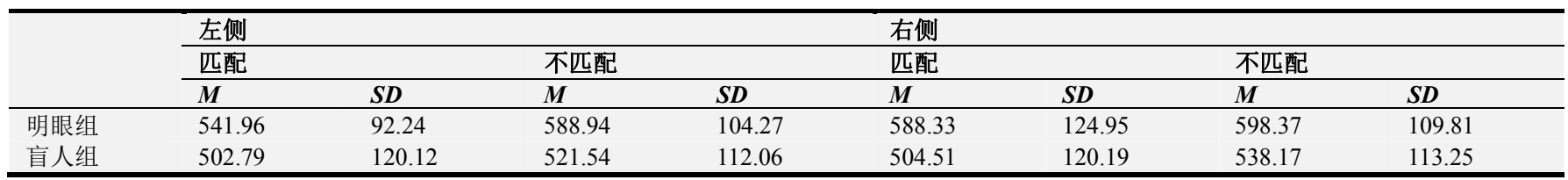

\section{4. 讨论}

本研究采用语义启动实验范式, 使用事件相关电位电 位技术, 测量并分析了先天盲被试和明眼被试在进行物体 颜色知识判断时的行为反应和电生理信号变化, 进而旨在 探讨视觉经验与颜色认知之间的关系。结果发现, 先天盲 被试对物体颜色判断的正确率高于随机水平, 但低于明眼 被试。两组被试中都有出现由颜色关系不匹配所诱发的 N400效应, 且这种N400效应在左侧电极更强, 表现出左
半球优势。但是, 先天盲被试的N400效应大于明眼被试的 N400效应, 先天盲被试N400反应的潜伏期小于明眼被试 $\mathrm{N} 400$ 反应的潜伏期。基于行为数据和脑电数据的分析结果, 下面, 本研究对视觉经验缺失对盲人颜色认知的影响进行 讨论。

行为反应分析结果显示, 盲人被试对物体颜色判断的 正确率 $(M=0.84)$ 高于随机水平, 说明他们具有一定的 物体颜色知识。人类使用语言来传递思想, 语言中包含大 量信息, 盲人可以通过与他人交流或阅读（盲文）等方式 获得颜色知识。但是, 盲人被试的正确率显著低于明眼被 
试 $(M=0.94)$ 。该结果启示我们, 直接的视觉感知体验 对颜色知识获得的重要作用, 视觉是获得物体颜色知识有 效的途径。另一方面, 盲人具有一定物体颜色知识的证据 说明, 人类对环境均有很强的适应能力, 当原有的知识获 取通道 (比如视觉) 受损时, 个体可能会发展出适应性的 学习策略, 通过其它完整的感觉通道 (比如听觉) 来进行 学习。因此, “感觉与认知相分离” [6-7, 13-14]和“认知基 于感觉” $[1,9,16]$ 这两种理论并不是对立的, 而是解释了 不同情况下的感觉和认知之间的关系。

脑电分析结果发现, 先天盲被试组和明眼被试组中均 有出现颜色不匹配所诱发的N400效应, 即两组被试中, 颜 色不匹配词对比颜色匹配词对所诱发的N 400 反应波幅更 大。并且, 不匹配条件下 $N 400$ 反应的潜伏期也长于匹配条 件下 400 反应的潜伏期。 N400反应可以表征存储于长时 记忆中的与目标词特征相关的一些表征的激活程度 [5], 当 启动颜色词与物体颜色匹配时, 与目标词相关的特征较容 易激活, 有利于目标词识别; 当启动颜色词与物体颜色不 匹配时, 个体则需要调动更多的认知资源来识别目标词, 因此不匹配条件下 $\mathrm{N} 400$ 反应的波幅更大、潜伏期更长。该 研究结果表明, 先天盲被试和明眼被试一样, 也能利用启 动词和目标词之间的颜色相关关系来促进他们判断。此外, 在盲人组和明眼组中均有发现, 左侧电极上的 $\mathrm{N} 400$ 效应 （N400反应差异波波幅）大于右侧电极上的N400效应, 显示两组被试物体颜色知识表征均具有左半球优势。该结 果获得最近一项关于盲人物体颜色知识的脑成像研究结 果的支持, Wang及其同事[17]发现先天盲被试和明眼被试 都有左半球背侧前题叶皮层支持其物体颜色知识表征。

本研究还发现, 先天盲被试 $\mathrm{N} 400$ 反应差异波波幅(即 N400效应）比明眼被试大, 并且, 先天盲被试N400反应 潜伏期比明眼被试短。直接的视觉感知体验为明眼人物体 颜色知识的习得提供了鲜明形象的线索, 先天盲个体不能 直接感知物体颜色, 只能通过语言描述来获得颜色知识。 日常生活中, 明眼人颜色知识的获得在有意或无意中无时 无刻都在进行着, 而盲人通过语言描述对颜色知识的获得 方式则具有一定局限性。这种获得途径的限制可能导致先 天盲个体的物体颜色知识弱于明眼人, 他们物体颜色知识 的认知空间可能小于明眼人。在进行物体颜色判断时, 可 能是因为先天盲被试所搜寻的认知空间较小, 他们更容易 对“匹配”和“不匹配”的关系进行认知分离, 因而盲人被试 组的 N400效应更大, N400反应潜伏期更短。

\section{5. 结论}

总而言之, 本研究发现, 先天盲被试具有一定的颜色 知识, 并且他们的颜色知识表征与明眼被试一样, 具有左 半球优势。但是, 先天盲被试的颜色知识水平比明眼被试 差, 先天盲被试中不匹配条件所诱发的 N400效应比明眼被 试大, N400反应的潜伏期比明眼被试短, 这可能是由于盲 人的颜色认知空间较小所致。

本究结果启示我们, 直接的视觉感知是明眼人获得物 体颜色知识的有效途径; 但是, 视觉经验缺失的盲人在认 知和神经生理方面产生了一些适应性改变一一先天盲人 使用与明眼人不同的策略来获得物体颜色知识, 并且, 他
们对物体颜色知识的认知神经表征也有一定影响。由此说 明, 视觉感知体验对个体的颜色认知的形成具有重要作用 但并非唯一途径。

\section{致谢}

本文研究得到了国家社会科学基金重大项目 (13\&ZD188) 和认知神经科学与学习国家重点实验室 2013年度开放课题（CNLYB1311）的经费支持。

\section{参考文献}

[1] Barsalou, L. W., Simmons, W. K., Barbey, A. K., \& Wilson, C. D. Grounding conceptual knowledge in modality-specific systems [J]. Trends in cognitive sciences, 2003, 7(2): 84-91.

[2] Kutas, M., \& Hillyard, S. A. Reading senseless sentences: Brain potentials reflect semantic incongruity [J]. Science, 1980, 207(4427): 203-205.

[3] Kutas, M., \& Iragui, V. The N400 in a semantic categorization task across 6 decades [J]. Electroencephalography and Clinical Neurophysiology/Evoked Potentials Section, 1998, 108(5): 456-471.

[4] Landau, B., Gleitman, L. R., \& Landau, B. Language and experience: Evidence from the blind child (Vol. 8) [M]. Harvard University Press, 2009.

[5] Lau, E. F., Phillips, C., \& Poeppel, D. A cortical network for semantics:(de) constructing the N400 [J]. Nature Reviews Neuroscience, 2008, 9(12): 920-933.

[6] Leshinskaya, A., \& Caramazza, A. For a cognitive neuroscience of concepts: Moving beyond the grounding issue [J]. Psychonomic bulletin \& review, 2016, 23(4): 991-1001.

[7] Mahon, B. Z., \& Caramazza, A. A critical look at the embodied cognition hypothesis and a new proposal for grounding conceptual content $[\mathrm{J}]$. Journal of physiology-Paris, 2008, 102(1-3): 59-70.

[8] Marmor, G. S. Age at onset of blindness and the development of the semantics of color names [J]. Journal of experimental child psychology, 1978, 25(2): 267-278.

[9] Martin, A. GRAPES - Grounding representations in action, perception, and emotion systems: How object properties and categories are represented in the human brain [J]. Psychonomic bulletin \& review, 2016, 23(4): 979-990.

[10] Mills, A. E. Language acquisition in the blind child: Normal and deficient [M]. San Diego, CA: College-Hill Press, 1983.

[11] Pérez-Pereira, M., \& Conti-Ramsden, G. Language development and social interaction in blind children [M]. Psychology Press, 2013.

[12] Saysani, A., Corballis, M. C., \& Corballis, P. M. Colour envisioned: Concepts of colour in the blind and sighted [J]. Visual Cognition, 2018, 26(5): 382-392.

[13] Shallice, T. Specialisation within the semantic system [J]. Cognitive neuropsychology, 1988, 5(1): 133-142. 
[14] Shallice, T. Impairments of semantic processing: Multiple dissociations $[\mathrm{J}]$. The cognitive neuropsychology of language, 2013, 111-128.

[15] Shepard, R. N., \& Cooper, L. A. Representation of colors in the blind, color-blind, and normally sighted [J]. Psychological Science, 1992, 3(2): 97-104.

[16] Simmons, W. K., Ramjee, V., Beauchamp, M. S., McRae, K., Martin, A., \& Barsalou, L. W. A common neural substrate for perceiving and knowing about color [J]. Neuropsychologia, 2007, 45(12): 2802-2810.
[17] Wang, X., Men, W., Gao, J., Caramazza, A., \& Bi, Y. Two Forms of Knowledge Representations in the Human Brain [J]. bioRxiv, 2019, 691931.

[18] 时琴琴. 盲童和正常儿童对颜色认知的对比 $[\mathrm{D}]$. 硕士学位 论文, 上海师范大学, 2011 .

[19] 张积家, 党玉晓, 章玉祉, 王惠萍, \& 罗观. 盲童心中的颜 色概念及其组织 [J]. 心理学报, 2008, 40(04): 389-401. 